\title{
The Impact of an Authentic Intervention on Students' Proportional Reasoning Skills
}

\author{
Mine Isiksal-Bostan1, Rukiye Ayan², Seçil Yemen-Karpuzcu ${ }^{3}$, Gamze Baktemur ${ }^{4}$ \\ Middle East Technical University, Turkey ${ }^{1,2,4}$ \\ Dumlupinar Unversity, Turkey ${ }^{2}$
}

\begin{abstract}
As a part of a long-term design-based research project, this study aimed to investigate the mean differences among the pre-, post- and retention tests of seventh grade students regarding their proportional reasoning skills. The data of this paper were collected from 30 seventh-grade students enrolled in a public school in Ankara, Turkey in the first and second semester of 2017-2018 academic year. Participants of the study were engaged in a teaching experiment aimed to develop their meaningful understanding of ratio and proportion unit through authentic activities. The intervention prolonged 30 lesson hours and Proportional Reasoning Test was applied to the students at the beginning of the instruction as pre-test, at the end of the instruction as post-test, and 5 months later as retention test. A one-way repeated measures of ANOVA was conducted to compare scores on the Proportional Reasoning Achievement Test at Time 1 (pretest), Time 2 (posttest) and Time 3 (retention test). Findings revealed that there was a significant difference among proportional reasoning skills. More specifically, there was a statistically significant increase in Proportional Reasoning Achievement Test scores from Time 1 to Time 2 but the difference between Time 2 and Time 3 was not statistically significant. Findings revealed the significant effect of intervention on students' proportional reasoning skills and retention of those skills. Based on the findings it could be deduced that designing authentic interventions could have an impact on enhancing and enduring students' proportional reasoning skills.
\end{abstract}

Index Terms - design-based research, middle school students, proportional reasoning, retention

\section{INTRODUCTION}

Proportional reasoning skills are important for many mathematical topics such as rational numbers [1]; fractions, percent, similarity, scaling, trigonometry [2]; algebra, geometry, problem solving [3]; probability and statistics [4]. In addition, proportional reasoning is essential for understanding many topics in science and situations in daily life [5], [6]. Furthermore, it is one of the most essential skills to be developed throughout middle school ( [7], [8]. Despite being such an essential skill, the instruction on proportional reasoning is still superficial in such a way that only missing value problems are given a place and the procedural aspects (i.e. cross multiplication) are emphasized during the instruction. However, it has been stressed that solving missing value problems with procedural algorithms cannot be regarded as an indicator of proportional reasoning [9].
Proportional reasoning is related to linking and iterating linked composites [10], understanding the multiplicative relationships in the proportional situations [11], comparing rates/ratio, [12] and qualitative reasoning [13]. Therefore, we conducted a design research study [14] in which we developed a series of authentic activities based on Realistic Mathematics Education [15] and implemented those activities in a seventh-grade classroom in two cycles. In this research, we hypothesize that seventh grade students' proportional reasoning skills could be enhanced through authentic activities.

\section{Methodology}

As a part of this long-term design research project, the purpose of this study is to investigate the mean differences among the pre-, post-, and retention tests of seventh grade students regarding their proportional reasoning skills. The data of this paper were collected from 30 seventh grade students enrolled in a public school in Ankara, Turkey in the first and second semester of 2017-2018 academic year. The data were collected through an achievement test at three times: Time 1-before the implementation, Time 2-right after the implementation, Time 3-five months later than the end of the implementation in order to investigate the mean differences among the pre-test, post-test and retention tests regarding their proportional reasoning skills. After the pretest, students were involved in activity-based instruction in which concepts regarding ratio and proportion were introduced through authentic activities. An example for the sample activity is given in the Appendix. Proportional reasoning achievement test was developed by the researchers and piloted with a seventh and an eighth-grade classes of students. A table of specification was prepared in order to ensure the test's validity for the five big ideas. The test included five parts, each of which was related to one of the five big ideas: linking composite units, iterating linked composites, multiplicative reasoning, comparing rates/ratios, and qualitative reasoning. The first two big ideas of linking composite units and iterating linked composites were measured through a context in which a rule for the link between the number of kids and candies was provided. Then, students were required to find whether or not there were enough candies for the given number of kids and the number of candies that would be needed for the given number of kids. A sample question is provided in Fig. 1 below. 

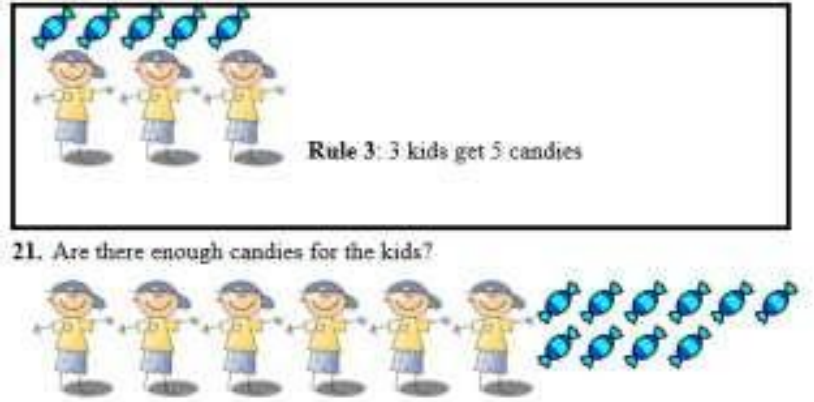

Fig. 1. A sample question in the test, which was used to measure linking and iterating composite units

The big idea of multiplicative reasoning was measured through short ratio tables including missing values of both integer and non-integer ratios. Students were required to reason about the invariant multiplicative relationship between the number of candies and kids and extend this reasoning to the second situation. A sample question that was designed to measure multiplicative reasoning is provided in Fig. 2 below.

\begin{tabular}{l|c|c}
$\begin{array}{l}\text { Number of } \\
\text { kids }\end{array}$ & $?$ & 9 \\
\hline $\begin{array}{l}\text { Number of } \\
\text { candies }\end{array}$ & 14 & 21
\end{tabular}

Fig. 2. A sample question in the test, which was used to measure multiplicative reasoning

The fourth big idea that was related to comparing ratios/rates was measured through a mixture problem, which included four pitchers including different amounts of lemon juice and water. Students were asked to determine the most orange-y mixture and justify their reasoning by comparing the amount of lemon juice and water in each pitcher. A sample question is provided in Fig. 3 below.

\begin{tabular}{|c|c|c|c|c|}
\hline & Pitcher A & Pitcher B & Pitcher C & Pitcher D \\
\hline & & & & \\
\hline Amount of lemon juice & 4 glasses & 2 glasses & 3 glasses & 5 glasses \\
\hline Amouat of water & 3 glasses & 2 glasses & 4 glasses & 5 glasses \\
\hline
\end{tabular}

\section{Which pitcher contains the most orange-y mixture? Explain}

Fig. 3. A sample question in the test, which was used to measure comparing ratios/rates

The last big idea that was qualitative reasoning was measured through a coffee and milk mixture in which students were asked to decide which of the cups would contain the stronger coffee taste. Even though this question was similar to the lemon juice question, this question did not include any numbers in it. Students were required to reason qualitatively without using numbers.

\section{RESULTS}

The data were examined based on students' total scores in general and students' scores in each big idea namely linking composite units, iterating linked composites, multiplicative reasoning, comparing rates/ratios, and qualitative reasoning. Table 1 shows the mean differences in each test for the total scores on the Proportional Reasoning Achievement Test at Time 1 (pre-test), Time 2 (post-test) and Time 3 (retention test).

The total scores are out of 100 points where linking composite units part is out of 12 points, iterating linked composites part is out of 32 points, multiplicative reasoning is out of 20 points and comparing rates/ratios and qualitative reasoning parts are out of 18 points. Findings revealed that there was an increase in post-test scores compared to the scores in the pre-test, but there was a slight decrease in the retention scores compared to the post-test scores.

Fig. 4. Mean differences of total scores in the proportional reasoning achievement test at the beginning, at the end of the instruction and five months later

The one-way repeated measures ANOVA results revealed that there was a statistically significant difference among proportional reasoning skills for total scores, Wilks' Lambda $=.000, \mathrm{~F}(2,28)=50.724, \mathrm{p}<.05$, partial eta squared $=.78$. The means and standard deviations are presented in Table 1 below.

TABLE I: DESCRIPTIVE STATISTICS FOR PROPORTIONAL REASONING ACHIEVEMENT TEST SCORES AT TIME 1, TIME 2 AND TIME 3 FOR TOTAL SCORES

\begin{tabular}{cccc}
\hline Test & $\mathrm{N}$ & Mean & SD \\
\hline Pre-test & 30 & 40.90 & 13.74 \\
Post-test & 30 & 69.13 & 20.23 \\
Retention & 30 & 66.14 & 24.56 \\
\hline
\end{tabular}

Three pair wise comparisons among the pair 1, pair 2 and pair 3 were conducted. The pair wise comparison in pair 1 (Proportional Reasoning Achievement Test scores between Time 1 and Time 2) and pair 3 (Proportional Reasoning 
Achievement Test scores between Time 1 and Time 3) were statistically significant at the .05 level. There was a statistically significant increase in Proportional Reasoning Achievement Test scores from Time 1 to Time 2 and from Time 1 to Time 3 . The eta squared statistics for pair 1 is .53, and for pair 3 is .38 , both of which indicating a large effect size. The pair wise comparison in pair 2 (Proportional Reasoning Achievement Test scores between Time 2 and Time 3) was not statistically significant at the .05 level.

Findings revealed the significant effect of intervention on students' proportional reasoning skills and retention of those skills in total scores and also in each of the five big ideas individually. More specifically, mean differences among the scores in pre-, post- and retention tests in terms of linking composite units, iterating linked composites, multiplicative reasoning, comparing rates/ratios, and qualitative reasoning is illustrated in Fig. 5 below.

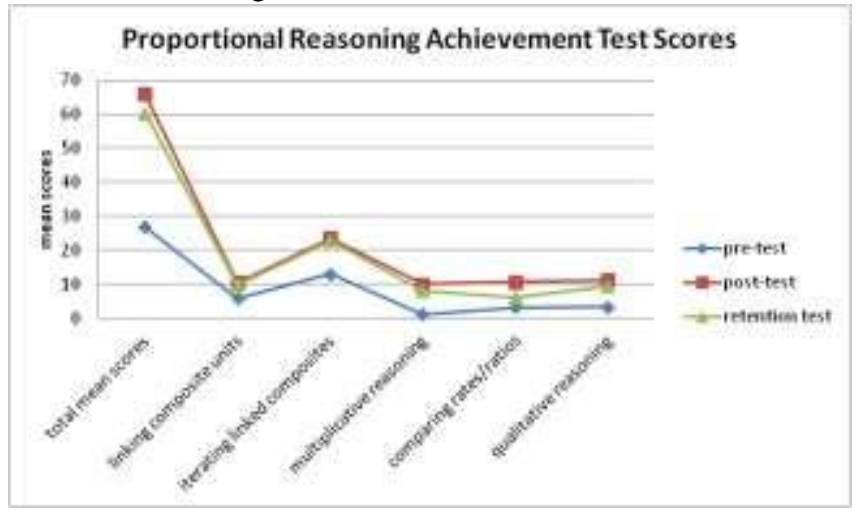

Fig. 5. Mean differences among the scores in three tests in terms of the five big ideas

As could be deduced from the above Figure, students' mean scores on the post-test is greater than their scores on the pre-test; but, there was a slight decrease in the retention scores compared to the post test scores. The one-way repeated measures ANOVA results revealed that there was a statistically significant mean difference among the scores in proportional reasoning skills achievement tests implemented at three times for linking composite units, iterating linked composites, multiplicative reasoning, comparing rates/ratios, and qualitative reasoning. Based on the quantitative results, it could be deduced that designing authentic interventions could have an impact on enhancing and enduring students' proportional reasoning skills.

\section{DISCUSSION AND CONCLUSION}

The goal of this study was to examine seventh grade students' enhancement of proportional reasoning skills from a quantitative point of view. For this purpose, we analyzed the mean differences in students' proportional reasoning skills before and after they were engaged in authentic tasks developed to enhance proportional reasoning skills. In addition, the retention of these skills was measured through the implementation of the same test five months after the end of the implementation. The one-way repeated measures of
ANOVA indicated that students' proportional reasoning skills were significantly increased after the implementation. Moreover, the retention of these skills was ensured. Therefore, our findings suggest that engaging students in authentic activities has a great potential in order to support and retain seventh grade students' proportional reasoning skills. In order to get in-depth explorations regarding the enhancement of proportional reasoning skills and in what ways these skills were supported, a qualitative research study could be conducted as a follow up. For instance, the discourse that took place as the students were engaged in authentic activities could be analyzed in order to see the ways students' proportional reasoning skills were enhanced.

\section{APPENDIX-A SAMPLE ACTIVITY}

The first activity is related to the link between the number of fish and food bars and the iteration of this link for different situations The activity involved five sequential parts that was developed so as to move students from informal ways of reasoning to more formal ways of reasoning. For instance, the first part included a one-to-many link (i.e. 1 food bar for 3 fish), which would allow students to work with an integer ratio. In addition, the tools to be used were sequenced from pictures and tables to more symbolic representations.. The activity was launched with a realistic story regarding the importance of feeding the fish with a certain amount of food bar in order to prevent under feeding or overfeeding. This story was developed to help students make sense of the link between the number of food bars and fish and why that link could not be broken.

In the first part of the activity, the rule is " 1 food bar feeds 3 fish, which includes 14 questions. The key ideas in the activity are for students to link two composite units, iterate these linked composites, determine the invariant multiplicative relationship and use build-up strategies. Students could link the two composite units by drawing pictures or tables, using numerical linking and iterating, or multiplying and dividing for making iterations. A sample question in the first part is provided in Fig. 6 below.

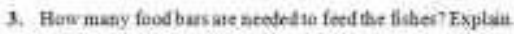

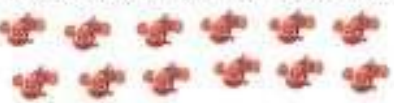 \\ Answer:}

Fig. 6. A sample question in the first part of the first activity

In the second part of the activity, the rule is " 1 food bar feeds 5 fish.", which includes 9 questions. The long ratio table is introduced as students deal with the questions in this part. The key learning ideas in this part of the activity is that students use horizontal relationships on the long ratio tables in order to keep track of iterations in addition to the strategies in the first part. A sample question in the second part is provided in Fig. 7 below. 


\section{How many food bars are needed to feed 55 fishes? Explain.}

Fig. 7. A sample question in the second part of the first activity

In the third part of the activity, the rule is " 2 food bars feed 4 fish", which includes 10 questions. The key learning idea in this part of the activity is that students make sense of the concept of unit rate and how working with unit rate makes computations easier. A sample question in the third part is provided in Fig. 8 below.

2. How many fishes can be fed with 8 food bars? Explain.

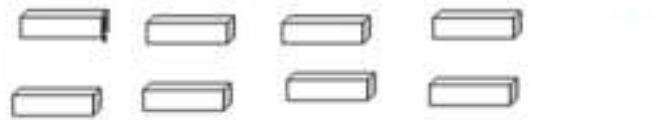

Fig. 8. A sample question in the third part of the first activity

In the fourth part of the activity, the rule is " 2 food bars feed three fish", which includes 9 questions. The key learning ideas in this part of the activity are that students use build-up and abbreviated build-up strategies in the long ratio tables and reason with a non-integer link ( 2 food bars for 3 fish) between the number of food bars and fish. A sample question in the fourth part is provided in Fig. 9 below.

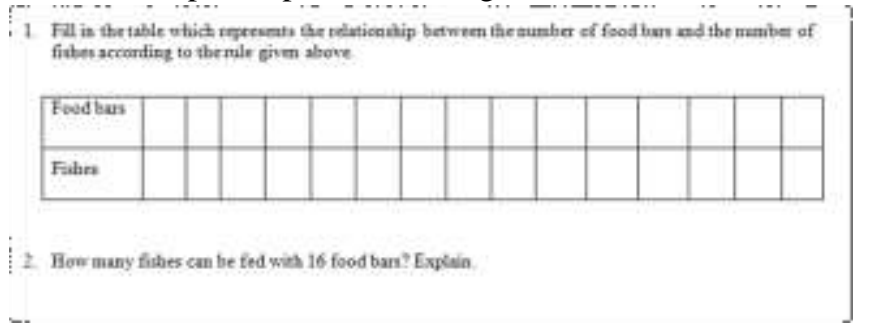

Fig. 9. A sample question in the fourth part of the first activity

In the fifth part of the activity, different rules regarding the link between the number of food bars and fish were given in each of the short ratio tables, which includes 4 questions. The key learning ideas in this part of the activity are that students switch to multiplicative thinking by shortening the long tables to short tables and use the invariant multiplicative reasoning between the number of food bars and fish to find the missing values in the tables. . A sample question in the fifth part is provided in Fig. 10 below.

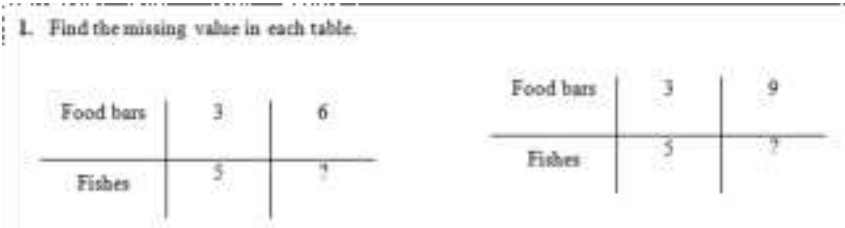

1. Use a takle to find the number of fiches that can be fed with 36 food hars

Fig. 10. A sample question in the fifth part of the first activity

\section{ACKNOWLEDGMENT}

This research is part of the research project $217 \mathrm{~K} 430$, funded by the Scientific and Technological Research Council of Turkey.

\section{REFERENCES}

[1] S. J. Lamon, "Ratio and proportion: connecting content and children's thinking," Journal for Research in Mathematics Education, vol. 24, no. 1, pp. 41-61, Jan. 1993.

[2] K. Beswick, "Make your own paint chart: a realistic context for developing proportional reasoning with ratios," Australian Mathematics Teacher, vol. 67, no. 1, pp. 6-11, 2011.

[3] S. B. Empson, "Equal sharing and shared meaning: The development of fraction concepts in a first-grade classroom," Cognition and Instruction, vol. 17, no. 3, pp. 283-342, 1999.

[4] C. Greenes and C. Fendell, Groundworks: Algebraic puzzles and problems, Chicago, IL: Creative Publications, 2000.

[5] R. Karplus, S. Pulos, and E. K. Stage, "Early adolescents' proportional reasoning on "rate" problems," Educational Studies in Mathematics, vol. 14, no. 3, pp. 219-233, 1983.

[6] A. G. Spinillo, and P. E. Bryant, "Proportional reasoning in young children: part-part comparisons about continuous and discontinuous quantity," Mathematical Cognition, vol. 5, no. 2, pp. 181-197,1999.

[7] Ministry of National Education [MoNE], Ortaokul matematik dersi (5, 6, 7 ve 8. Sinıflar) ögretim programı [Middle school mathematics curricula for grades 5, 6, 7, and 8]. Ankara, Turkey: MEB, 2013.

[8] National Council of Teachers of Mathematics [NCTM], Curriculum and evaluation standards for school mathematics. Reston, VA: The Council, 1989.

[9] R. Lesh, T. Post, and M. Behr, "Proportional reasoning," in Number concepts and operations in the middle grades, J. Hiebert and M. Behr, Eds. Reston, VA: Lawrence Erlbaum \& National Council of Teachers of Mathematics, 1988, pp. 93-118.

[10] M. T. Battista and C. Van Auken Borrow, "A proposed constructive itinerary from iterating composite units to ratio and proportion concepts," presented at the annual meeting of the North American Chapter of the International Group for Psychology of Mathematics Education, Columbus, OH, October, 1995.

[11] K. Cramer, and T. Post, "Making connections: A case for proportionality," Arithmetic Teacher, vol. 60, no. 6, pp. 342-346, 1993.

[12] G. Noelting, "The development of proportional reasoning and the ratio concept part I- Differentiation of stages," Educational Studies in Mathematics, vol. 11, no. 2, pp. 217-253, 1980.

[13] M. Behr, G. Harel, T. Post, and R. Lesh. "Rational number, ratio and proportion," in Handbook on research of teaching and learning, D. Grouws Ed. New York, NY: McMillan. 1992, pp. 296-333.

[14] K. Gravemeijer, and P. Cobb, "Design research from a learning design perspective" in Educational design research, J. Van den Akker, K Gravemeijer, S. McKenney, and N. Nieveen Eds. London, England: Routledge. 2006, pp. 17-51.

[15] K. Gravemeijer, "How emergent models may foster the constitution of formal mathematics". Mathematical Thinking and Learning, vol. 1, no. 2, pp. $155-177,1999$ 